\title{
Myocarditis in a patient treated with Nivolumab and PROSTVAC: a case report
}

\footnotetext{
* Correspondence: marijo.bilusic@nih.gov

${ }^{4}$ Genitourinary Malignancies Branch, Center for Cancer Research, National

Cancer Institute, National Institutes of Health, 10 Center Dr., Rm. B2L312,

Bethesda, MD 20892, USA

Full list of author information is available at the end of the article

(c) The Author(s). 2018 Open Access This article is distributed under the terms of the Creative Commons Attribution 4.0 International License (http://creativecommons.org/licenses/by/4.0/), which permits unrestricted use, distribution, and reproduction in any medium, provided you give appropriate credit to the original author(s) and the source, provide a link to the Creative Commons license, and indicate if changes were made. The Creative Commons Public Domain Dedication waiver (http://creativecommons.org/publicdomain/zero/1.0/) applies to the data made available in this article, unless otherwise stated.
}

\begin{abstract}
Background: Immune checkpoint inhibitors have revolutionized treatment and improved survival in many cancers. mortality if not recognized and treated early. (mCRPC) treated with a combination of nivolumab, an anti-programmed cell death protein 1 antibody, and PROSTVAC, a vector-based therapeutic prostate cancer vaccine.

Case Report: A 79-year-old man with MCRPC metastatic to bone and lymph nodes and a history of atrial fibrillation

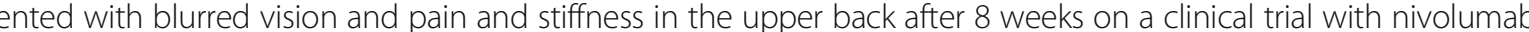
$0.209 \mathrm{ng} / \mathrm{mL}$ (normal: 0-0.056 ng/mL). Electrocardiogram (ECG) revealed atrial fibrillation with QT prolongation (QTc (45 mmHg). ProBNP was elevated at $1463 \mathrm{pg} / \mathrm{mL}$ and peaked at $3066 \mathrm{pg} / \mathrm{mL}$ one day after hydration. With a presumed diagnosis of autoimmune myositis and possible myocarditis, the patient was admitted and started on myocarditis (Fig. 1). The patient was discharged on a prednisone taper after normalization of cardiac enzymes on day 4. Treatment with PROSTVAC continued for three more months; nivolumab was discontinued. Six months later, patient is doing well, with no residual cardiac damage.


(Continued from previous page)

Discussion: Cardiovascular irAEs are relatively rare $(<1 \%)$ and have a variety of clinical presentations. Myocarditis is potentially life-threatening and can range from subclinical to fulminant. Therefore, clinical suspicion, early detection, and prompt treatment are imperative (1). The initial diagnostic workup should include cardiac enzymes, ECG, and 2Dechocardiogram. The most commonly observed ECG changes are generalized repolarization abnormalities, prolonged QT interval, and conduction abnormalities (2). An elevated troponin I in the absence of overt coronary artery disease is suggestive of myocarditis and should be evaluated further. Myocardial biopsy is the standard diagnostic procedure; however, a cardiac MRI can achieve a diagnosis when biopsy is not feasible (3). Advancements in parametric mapping techniques have allowed the use of native myocardial T1 in the detection of myocarditis, as it has superior diagnostic performance and higher sensitivity than older parameters (3). Our patient had been treated with an immune checkpoint inhibitor and a therapeutic cancer vaccine to induce effective antitumor activity through immunogenic intensification and presented with muscle stiffness and elevated CK. Although he had no new cardiovascular symptoms, cardiac enzymes were tested to rule out myocardial involvement. MRI with gadolinium confirmed the diagnosis of myocarditis. To date, none of the 1360 patients treated with PROSTVAC as a single agent have developed myocarditis, while myocarditis has been rarely reported in patients treated with nivolumab $(<1 \%)(1)$. Whether the combination of PROSTVAC and nivolumab presents an additional risk of myocarditis is unclear. To our knowledge, this is the first case of myocarditis in a patient with $\mathrm{mCRPC}$ receiving simultaneous treatment with an immune checkpoint inhibitor and a prostate cancer vaccine.

Our experience highlights the importance of suspicion and early intervention in patients who present with cardiac abnormalities after receiving cancer immunotherapy. We propose following protocol: baseline troponin, ECG, and 2D-echocardiogram prior to treatment, then repeated troponin at 2, 4, and 12 weeks post-treatment, then monthly. If troponin becomes positive without alternative explanation, myocarditis should be ruled out with cardiac MRI or myocardial biopsy, and patient should be admitted for treatment with high-dose steroids as early intervention may minimize myocardial injury.

\section{Background}

Immune checkpoint inhibitors have revolutionized treatment and improved survival in many cancers. However, since immune-related adverse events (irAEs) are potentially fatal, early recognition and prompt treatment are warranted. Immune myocarditis exemplifies the significance of such a need highlighting the importance of raising the awareness of the risk and condition that will lead to an expedited intervention.

\section{Objective}

To report the first case of myocarditis in a patient with metastatic castration-resistant prostate cancer (mCRPC) treated with a combination of nivolumab, an anti-programmed cell death protein 1 antibody, and PROSTVAC, a vector-based therapeutic prostate cancer vaccine targeting prostate specific antigen (PSA).

\section{Case report}

A 79-year-old man with mCRPC metastatic to bone and lymph nodes and a history of atrial fibrillation presented with blurred vision and pain and stiffness in the upper back after 8 weeks on a clinical trial with nivolumab $(1 \mathrm{mg} / \mathrm{kg})$ and PROSTVAC, both given every 2 weeks. Eye exam was within normal limits, while musculoskeletal exam revealed tenderness in trapezius muscles and decreased motor strength in arms (III/V) and neck (I V/V). The rest of the physical exam was within normal limits, with the exception of an irregular heart rhythm. Laboratory tests were as follows: creatinine kinase (CK) 3200 U/L (normal: 39-308 U/L), CK-MB $65.7 \mathrm{mcg} / \mathrm{L}$ (normal: 0-7.6 mcg/L), troponin I $0.209 \mathrm{ng} / \mathrm{mL}$ (normal: $0-0.056 \mathrm{ng} / \mathrm{mL}$ ). Electrocardiogram (ECG) revealed atrial fibrillation with QT prolongation (QTc $514 \mathrm{msec}$ ) and left anterior fascicular block, all unchanged from baseline three months before. 2D-echocardiogram showed a left ventricular ejection fraction of $65 \%$ with an enlarged left atrium, a dilated right ventricle and increased pulmonary artery pressure $(45 \mathrm{mmHg})$. ProBNP was elevated at $1463 \mathrm{pg} / \mathrm{mL}$ and peaked at $3066 \mathrm{pg} / \mathrm{mL}$ one day later after hydration. With a presumed diagnosis of autoimmune myositis and possible myocarditis, the patient was admitted and started on methylprednisolone 1 $\mathrm{mg} / \mathrm{kg} /$ day.

The cardiac MRI findings were consistent with myocarditis involving small areas of the myocardium. Left ventricular size and function were normal (ejection fraction 59\%) with normal regional wall motion. Patches of late gadolinium enhancement (LGE) were seen in the basal and mid inferior wall showing an epicardial pattern compatible with myocarditis. Early gadolinium enhancement was abnormal in a similar distribution to the LGE but more extensive. Myocardial T1 was $1411 \mathrm{~ms}$. in the basal inferoseptum and $1231 \mathrm{~ms}$. in the mid inferior wall (normal native $\mathrm{T} 1<1350 \mathrm{~ms}$.). 
Myocardial T2 was normal in all segments except the mid inferior wall where it was at the borderline between normal and mildly elevated. Extra cellular volume fraction (ECV) was more diffusely abnormal in the basal inferior wall 41.1\% (normal 25.5, 95\% confidence intervals 20.5$30.5 \%$ ) in the closest region of interest to the area of abnormal LGE but also diffusely mildly elevated (32-33\%) in several basal and mid ventricular segments (Fig. 1).

The patient was discharged on a multiple week oral prednisone taper after normalization of cardiac enzymes on day 4. Treatment with PROSTVAC continued for three more months; nivolumab was discontinued. Six months later, patient is doing well, with no residual cardiac damage.

\section{Discussion}

Cardiovascular irAEs are uncommon (approximately 1\%) and have a variety of clinical presentations. Myocarditis is potentially life-threatening and can range from subclinical to fulminant. Therefore, clinical suspicion, early detection, and prompt treatment are imperative [1-3]. The initial diagnostic workup should include cardiac enzymes, ECG, and 2D-echocardiogram. The most commonly observed ECG changes are generalized repolarization abnormalities, prolonged QT interval, and conduction abnormalities [4]. An elevated troponin I in the absence of overt coronary artery disease is suggestive of myocarditis and should be evaluated further. Myocardial biopsy is the standard diagnostic procedure; however, a cardiac MRI can achieve a diagnosis when biopsy is not feasible. Cardiac MRI imaging in conjunction with the Lake Louis Criteria have a sensitivity of $74 \%$ and a specificity of $86 \%$ [5]. Our patient had been treated with an immune checkpoint inhibitor and a therapeutic cancer vaccine to induce effective antitumor activity through immunogenic intensification and presented with muscle stiffness and elevated $\mathrm{CK}$. Although he had no new cardiovascular symptoms, cardiac enzymes were tested to rule out myocardial involvement. MRI with gadolinium confirmed the diagnosis of myocarditis. To date, none of the 1360 patients treated with PROSTVAC as a single agent have developed myocarditis, while myocarditis has been reported in patients treated with nivolumab (approximately 1\%) [1, 3]. Whether the combination of PROSTVAC and nivolumab presents an additional risk of myocarditis is unclear. To our knowledge, this is the first case of myocarditis in a patient with $\mathrm{mCRPC}$ receiving simultaneous treatment

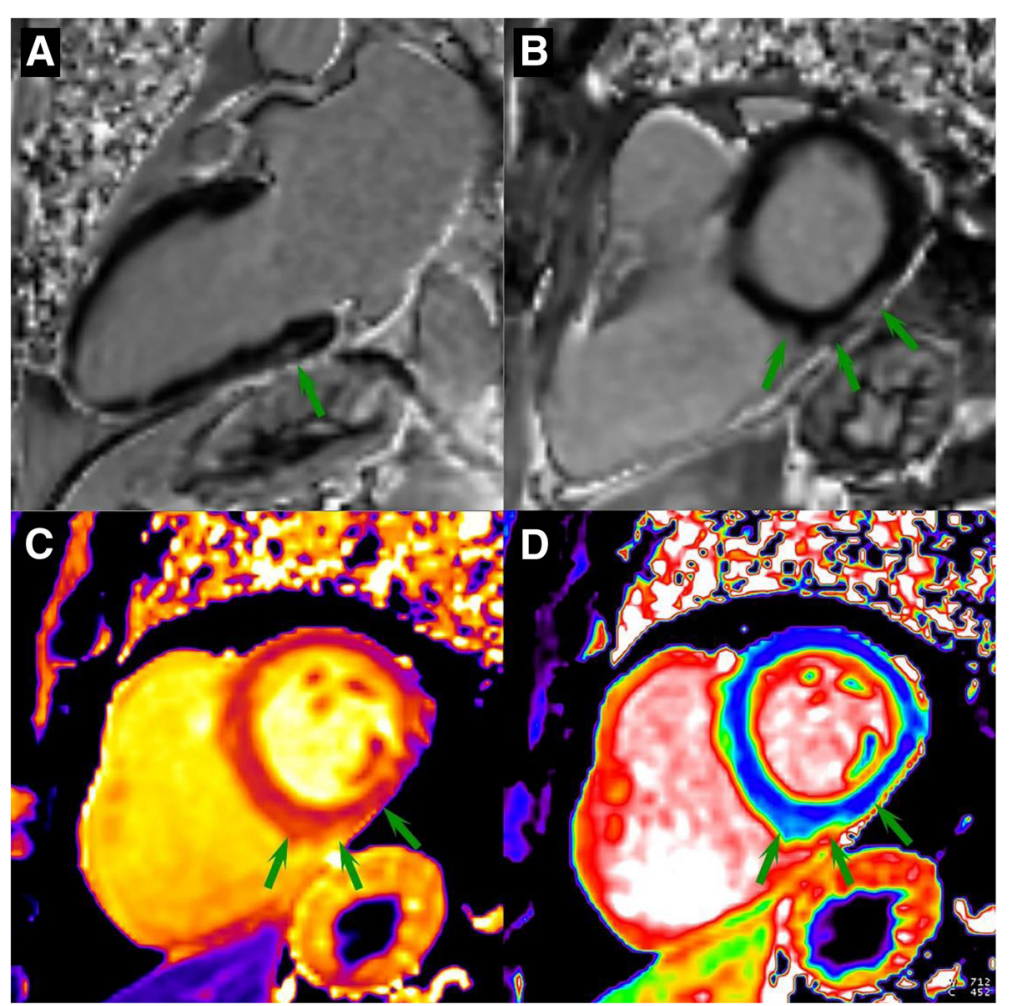

Fig. 1 CMR findings support a diagnosis of myocarditis. Late gadolinium enhancement (LGE) images in long axis (a) and short axis (b) views show epicardial enhancement in the basal inferior wall and inferior septum (arrows). Native T1 (c) and extracellular volume fraction (d) were abnormal in the basal inferior wall and inferior septum. Mid inferior myocardial T2 was at the borderline between normal and mildly abnormal (not show). Early gadolinium enhancement (not shown) was also abnormal in a similar but more extensive distribution than the LGE 
with an immune checkpoint inhibitor and a prostate cancer vaccine.

Our experience highlights the importance of suspicion, diagnosis and early intervention in patients who present with cardiac abnormalities and possible myocarditis after receiving immunotherapy [6]. Prompt treatment with high-dose steroids may minimize myocardial injury.

Acknowledgments

Not applicable.

\section{Funding}

This manuscript did not entail any funding sources outside of our institution.

\section{Availability of data and materials}

Not applicable.

\section{Disclaimer/disclosures}

None

\section{Authors' contributions}

All authors contributed equally to this case report. All authors read and approved the final manuscript.

\section{Ethics approval and consent to participate}

Ethics approval waived in case reports.

\section{Consent for publication}

Consent for publication form has been obtained.

\section{Competing interests}

The authors declare that they have no competing interests.

\section{Publisher's Note}

Springer Nature remains neutral with regard to jurisdictional claims in published maps and institutional affiliations.

\section{Author details}

${ }^{1}$ Medical Oncology Service, Center for Cancer Research, National Cancer Institute, National Institutes of Health, Bethesda, MD, USA. ${ }^{2}$ Vaccine Branch, Center for Cancer Research, National Cancer Institute, National Institutes of Health, Bethesda, MD, USA. ${ }^{3}$ Cardiovascular Branch, National Heart, Lung, and Blood Institute, National Institutes of Health, Bethesda, MD, USA.

${ }^{4}$ Genitourinary Malignancies Branch, Center for Cancer Research, National Cancer Institute, National Institutes of Health, 10 Center Dr., Rm. B2L312, Bethesda, MD 20892, USA.

Received: 4 August 2018 Accepted: 3 December 2018

Published online: 18 December 2018

\section{References}

1. Johnson DB, Balko JM, Compton ML, Chalkias S, Gorham J, Xu Y, et al. Fulminant myocarditis with combination immune checkpoint blockade N Engl J Med 2016:375:1749-1755. [PMID 27806233].

2. Moslehi JJ, Salem JE, Sosman JA. Increased reporting of fatal immune checkpoint inhibitor associated myocarditis. Lancet. 2018; 391:933.

3. Mahmood SS, Fradley MG, Cohen JV, Anju Nohria KL, et al. Myocarditis in patients treated with immune checkpoint inhibitors. J. Am. Coll. Cardiol. 2018. https://doi.org/10.1016/j.jacc.2018.02.037.

4. Wang DY, Okoye GD, Neilan TG, Johnson DB, Moslehi JJ. Cardiovascular toxicities associated with Cancer immunotherapies. Curr Cardiol Rep 2017; 19:21. [PMID 28220466].

5. Pan JA, Yoo JL, Salerno M. Diagnostic performance of extracellular volume, native T1, and T2 mapping versus Lake Louise criteria by cardiac magnetic resonance for detection of acute myocarditis. Circ
Cardiovasc Imaging. 2018;11:e007598. https://doi.org/10.1161/ CIRCIMAGING.118.007598.

6. Tay RY, Blackley E, McLean C, Moore, M. Succesful use of equine antithymocyte globulin (ATGAM) for fulminant myocardiutis secondary to nivolumab therapy. Br J Cancer2017;117 (7):921-924. https://doi.org/10. 1038/bjc.2017.253. Epub 2017 Aug10.
Ready to submit your research? Choose BMC and benefit from:

- fast, convenient online submission

- thorough peer review by experienced researchers in your field

- rapid publication on acceptance

- support for research data, including large and complex data types

- gold Open Access which fosters wider collaboration and increased citations

- maximum visibility for your research: over $100 \mathrm{M}$ website views per year

At BMC, research is always in progress.

Learn more biomedcentral.com/submission 